\title{
Evolution of the Higgs mode in a fermion superfluid with tunable interactions
}

\author{
Boyang Liu, ${ }^{1}$ Hui Zhai, ${ }^{1}$ and Shizhong Zhang ${ }^{2}$ \\ ${ }^{1}$ Institute for Advanced Study, Tsinghua University, Beijing 100084, China \\ ${ }^{2}$ Department of Physics and Center of Theoretical and Computational Physics, The University of Hong Kong, Hong Kong, China
}

(Received 27 January 2016; published 23 March 2016)

\begin{abstract}
In this work we present a coherent picture for the evolution of Higgs mode in neutral $s$-wave fermion superfluids. As the strength of attractive interaction between fermions increases from the BCS to the Bose-Einstein condensate (BEC) regime we find that the Higgs mode in the fermionic superfluids is pushed to higher energy while at the same time, gradually loses its spectral weight, which is because the system is tuned away from Lorentz invariance. Furthermore, when damping is taken into account, the spectral weight of the Higgs mode is significantly broadened due to coupling to phase mode in the whole BEC-BCS crossover.
\end{abstract}

DOI: 10.1103/PhysRevA.93.033641

\section{INTRODUCTION}

The experimental search for the Higgs boson in particle physics has made remarkable progress $[1,2]$. On the other hand, the Higgs mode has also generated considerable interest in condensed-matter and cold-atom systems. In the early 1980s, Raman scattering experiment revealed an unexpected peak in a superconducting charge density wave compound $\mathrm{NbSe}_{2}$ [3], which was later attributed to the Higgs mode [4,5]. The signal of the Higgs mode has also been observed in antiferromagnet $\mathrm{TlCuCl}_{3}$ by neutron scattering [6], and recently in a superconducting $\mathrm{NbN}$ sample by terahertz pump probe spectroscopy in a nonadiabatic excitation regime [7,8]. In cold-atom systems, the Higgs mode has been observed near the superfluid-to-Mott insulator phase transition of bosonic atoms in optical lattices at integer filling $[9,10]$.

Theoretically, the simplest field theory where the Higgs mode emerges is a relativistic U(1) field theory with Lorentz invariance in the symmetry-broken phase. This occurs, for example, in the weak-coupling BCS superconductor [11,12] or in the Mott-superfluid transition of the Bose-Hubbard model at integer filling [13,14]. However, in most condensed-matter systems, Lorentz invariance emerges only with fine tuning and the generic symmetry is usually Galilean [15]. Thus, it is an interesting question to investigate how the Higgs mode evolves as the system is tuned away from the Lorentz invariance point. Moreover, in condensed-matter systems, further complications often occur because the Higgs mode is usually coupled to other elementary excitations, which leads to its damping [16-19].

In this work, we investigate these issues in the context of the BEC-BCS crossover model. In the BCS limit, the system obeys approximate Lorentz symmetry due to particle-hole symmetry and is expected to host the Higgs mode. In the BEC limit, it is a condensate of molecular bosons and obeys the Galilean invariance. Here arises the first question that we would like to address, namely, what is the fate of the Higgs mode as the system is tuned away from the Lorentz-invariant limit. Second, due to tunable interactions in the BEC-BCS crossover, interaction effects on the Higgs mode due to coupling to collective and quasiparticle excitations can be thoroughly investigated [20]. In the following, we shall address these two aspects of the problem in turn.
The paper is organized as follows. In Sec. II, we discuss the theoretical framework that we employ to address the problem, namely, the time-dependent Ginzburg-Landau (TDGL) formulation of BEC-BCS crossover close to the transition temperature. We emphasize that in order to address the Lorentz-invariant limit, we need to extend the theory to include the second-order time-derivative term which is usually neglected in the context of BEC-BCS crossover [21]. In Sec. III, we discuss the effects of breaking Lorentz invariance when the system approaches the BEC limit. We discuss how the spectral weight of the Higgs mode is increasingly transferred to the phase (Goldstone) mode. In Sec. IV, we include the effects of damping on the spectral function of the Higgs mode (its frequency and spectral weight). We show that in addition to the usual broadening of the spectral function for the Higgs mode, its frequency is also shifted due to damping. Finally, we give a brief conclusion in Sec. V.

\section{TIME-DEPENDENT GINZBURG-LANDAU THEORY}

Close to the superfluid transition temperature, the BECBCS crossover can be conveniently formulated in terms of a Ginzburg-Landau energy functional. To include the effects of temporary fluctuations, time-dependent terms are required [21]. For our purposes, it is necessary to include the secondorder time-derivative term. Let us then write the action as

$$
S=\int d t d^{3} \mathbf{x}\left[\phi^{*}\left(-i u \partial_{t}+v \partial_{t}^{2}-\frac{\nabla^{2}}{2 m^{*}}-r\right) \phi+\frac{b}{2}|\phi|^{4}\right],
$$

where $\phi$ is the Ginzburg-Landau order parameter. The various parameters $u, v, r, b$, and $m^{*}$ can be computed along BEC-BCS crossover in terms of the chemical potential $\mu$, temperature $T$, and interaction parameter $\zeta=1 /\left(k_{\mathrm{F}} a_{\mathrm{s}}\right)$, where $a_{\mathrm{s}}$ is the $s$-wave scattering length. In this work, we use the Nozières-Schmitt-Rink (NSR) [22] scheme to compute these parameters, as detailed in the Appendix. The coefficients of the time-derivative terms $u=u^{\prime}+i u^{\prime \prime}$ and $v=v^{\prime}+i v^{\prime \prime}$ are complex in general. The real parts $u^{\prime}$ and $v^{\prime}$ describe the propagating behavior of the Cooper pair field, while the imaginary parts $u^{\prime \prime}$ and $v^{\prime \prime}$ describe its damping due to coupling to the fermionic quasiparticles. A plot of various parameters is given in Fig. 1. We note the following features: 


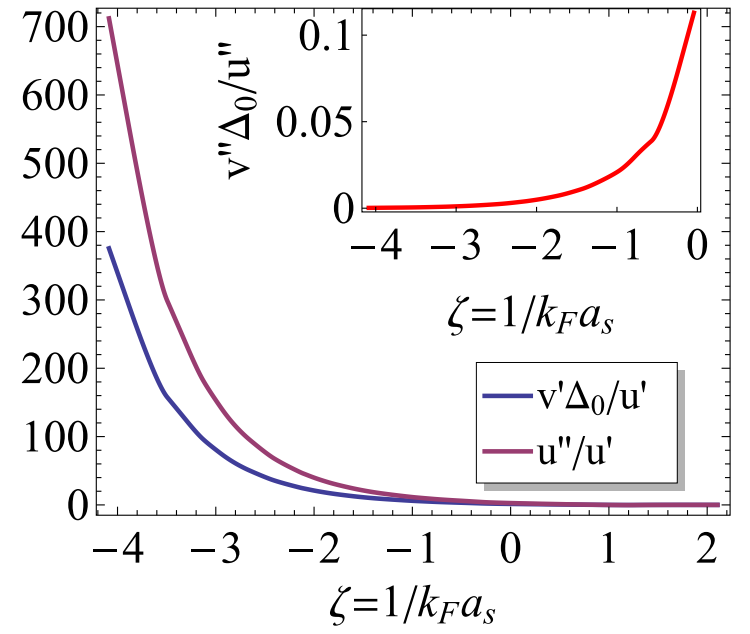

FIG. 1. $v^{\prime} \Delta_{0} / u^{\prime}$ and $u^{\prime \prime} / u^{\prime}$ as functions of the parameter $\zeta=$ $1 / k_{F} a_{s}$, where $k_{F}$ is the Fermi wave vector and $a_{s}$ is the $s$-wave scattering length. In the inset we show $v^{\prime \prime} \Delta_{0} / u^{\prime \prime}$ as a function of $\zeta$.

(i) Consider the real parts $u^{\prime}$ and $v^{\prime}$ in the BEC-BCS crossover. In the BCS limit, $u^{\prime} / v^{\prime} \Delta_{0} \rightarrow 0$ because of the approximate particle-hole symmetry in the weak-coupling BCS theory, while $\Delta_{0}=\sqrt{r / b}$ is the mean-field value of the order parameter. As a result, the system acquires an emergent Lorentz invariance, and one expects the emergence of Higgs mode, together with the standard phase (Goldstone or Anderson-Bogoliubov) mode for neutral fermion superfluid. In the BEC limit, however, $v^{\prime} \Delta_{0} / u^{\prime} \sim \Delta_{0} /|\mu| \ll 1$, and we can neglect the $v^{\prime}$ term. This leads to a Galilean-invariant neutral boson theory, for which only the Bogoliubov mode exists.

(ii) The damping terms $\left(u^{\prime \prime}\right)$ become important as one moves to the BCS side because of the decreasing fermionic excitation gap, and as a result, a stronger coupling of the pairing field to the quasiparticle excitations. This corresponds to a finite lifetime of Cooper pairs at finite temperature. We will show that the damping $u^{\prime \prime}$ term itself will generate a considerable effect for the appearance of the Higgs mode different from that in a pure Lorentz invariance theory. In the BEC limit, the imaginary parts vanish within the NSR. On the other hand, we find that whenever they are nonzero, $v^{\prime \prime} \Delta_{0} / u^{\prime \prime} \ll 1$ for the entire crossover regime and we shall thus neglect the $v^{\prime \prime}$ term altogether in the following discussion.

\section{EVOLUTION OF THE HIGGS MODE FROM THE BEC TO BEC LIMIT WITHOUT DAMPING-EFFECT OF BREAKING LORENTZ INVARIANCE}

To investigate the evolution of the Higgs mode as the system is gradually tuned from its Lorentz-invariant BCS limit towards the Galilean-invariant BEC limit, we shall first neglect the damping terms in Eq. (1) and study the transfer of spectral weight between the Higgs and phase modes. In the symmetry-broken state, we can write the order parameter $\phi=$ $\Delta_{0}+\delta_{a}+i \delta_{p}$, where $\delta_{a}$ and $\delta_{p}$ describe amplitude and phase fluctuations, respectively. In terms of $\delta_{a}$ and $\delta_{p}$ and with $u^{\prime \prime}=$ $v^{\prime \prime}=0$, we can write the action equation (1) in the Fourier space as

$$
S=\int \frac{d \omega}{2 \pi} \frac{d^{3} \mathbf{k}}{(2 \pi)^{3}} \bar{\Phi}(-\omega,-\mathbf{k}) \mathcal{G}^{-1} \Phi(\omega, \mathbf{k}),
$$

with $\bar{\Phi}(\omega, \mathbf{k})=\left[\delta_{a}(\omega, \mathbf{k}), \delta_{p}(\omega, \mathbf{k})\right]$, and the kernel $\mathcal{G}$ is given by

$$
\mathcal{G}^{-1}=\left(\begin{array}{cc}
-v^{\prime} \omega^{2}+\xi_{\mathbf{k}}+2 r & i u^{\prime} \omega \\
-i u^{\prime} \omega & -v^{\prime} \omega^{2}+\xi_{\mathbf{k}}
\end{array}\right)
$$

where $\xi_{\mathbf{k}}=k^{2} / 2 m^{*}$. Then the amplitude-amplitude correlation function can be easily calculated as

$$
\begin{aligned}
\mathcal{G}_{a a}(\omega, \mathbf{k}) & =\left\langle\delta_{a}(\omega, \mathbf{k}) \delta_{a}(-\omega,-\mathbf{k})\right\rangle \\
& =\frac{-v^{\prime} \omega^{2}+\xi_{\mathbf{k}}}{-u^{\prime 2} \omega^{2}+\left(-v^{\prime} \omega^{2}+\xi_{\mathbf{k}}\right)\left(-v^{\prime} \omega^{2}+\xi_{\mathbf{k}}+2 r\right)} .
\end{aligned}
$$

Straightforward calculation yields the spectral function of the Higgs mode as

$$
\begin{aligned}
A_{a a}(\omega, \mathbf{k}) & =-\frac{1}{\pi} \operatorname{Im} \mathcal{G}_{a a}(\omega+i \delta, \mathbf{k}) \\
& =A_{+}(\mathbf{k}) \delta\left(\omega-\omega_{+}(\mathbf{k})\right)+A_{-}(\mathbf{k}) \delta\left(\omega-\omega_{-}(\mathbf{k})\right) .
\end{aligned}
$$

It consists of two $\delta$ peaks at mode frequencies $\omega_{ \pm}(\mathbf{k})$ given by

$$
\omega_{ \pm}^{2}=\frac{\xi_{\mathbf{k}}+r}{v^{\prime}}+\frac{u^{\prime 2}}{2 v^{\prime 2}} \pm \sqrt{\frac{r^{2}}{v^{\prime 2}}+\frac{u^{\prime 4}}{4 v^{\prime 4}}+\frac{u^{\prime 2}}{v^{\prime 3}}\left(\xi_{\mathbf{k}}+r\right)},
$$

and the spectra densities at $\omega_{ \pm}$are given by

$$
\begin{aligned}
& A_{+}(\mathbf{k})=\frac{v^{\prime} \omega_{+}^{2}-\xi_{\mathbf{k}}}{2 v^{\prime 2} \omega_{+}\left(\omega_{+}^{2}-\omega_{-}^{2}\right)}, \\
& A_{-}(\mathbf{k})=\frac{-v^{\prime} \omega_{-}^{2}+\xi_{\mathbf{k}}}{2 v^{\prime 2} \omega_{-}\left(\omega_{+}^{2}-\omega_{-}^{2}\right)} .
\end{aligned}
$$

In the BCS limit, $v^{\prime} \Delta_{0} \gg u^{\prime}$ and solutions can be approximated as $\omega_{-}(k) \simeq k / \sqrt{2 m^{*} v^{\prime}}$ and $\omega_{+}(k) \simeq \sqrt{\left(\xi_{\mathbf{k}}+2 r\right) / v^{\prime}}$; the first being the phase (Goldstone) mode with linear dispersion and the second is the Higgs mode, with Higgs gap $\omega_{+}(0)=\sqrt{2 r / v^{\prime}}=\sqrt{2 b \Delta_{0}^{2} / v^{\prime}}$. Using the facts that $v^{\prime}=$ $7 \beta^{2} \zeta(3) v\left(\epsilon_{F}\right) / 16 \pi^{2}$ and $b=7 \beta^{2} \zeta(3) \nu\left(\epsilon_{F}\right) / 8 \pi^{2}$ in the BCS limit (see Appendix), one finds $\omega_{+}(0)=2 \Delta_{0}$, as expected for a Lorentz-invariant theory. Here $\beta=1 / k_{B} T$ is the inverse temperature, $\zeta(n)$ is the Riemann $\zeta$ function, and $v\left(\epsilon_{F}\right)=(2 m)^{3 / 2} \sqrt{\epsilon_{F}} / 4 \pi^{2}$ is the density of state at the Fermi energy $\epsilon_{F}$.

On the other hand, in the BEC limit, one finds that $u^{\prime} \gg v^{\prime} \Delta_{0}$, and $\omega_{-}(k)=\sqrt{\xi_{\mathbf{k}}\left(\xi_{\mathbf{k}}+2 r\right)} / u^{\prime}$ is the phase (Bogoliubov) mode while the other mode $\omega_{+}(k)=$ $\sqrt{2 \xi_{\mathbf{k}} / v^{\prime}+2 r / v^{\prime}+\left(u^{\prime} / v^{\prime}\right)^{2}}$ has a gap $\sim|\mu|$, of order of binding energy of the molecule in the BEC limit. The existence of the gapped mode is a reflection of the fact that our bosonic field $\phi$ is a composite of two fermions and disappears in the infinite binding limit where only the phase (Bogoliubov) mode exists as it should.

In between these two limits, Lorentz invariance is broken and the coupling between the amplitude and phase degrees 

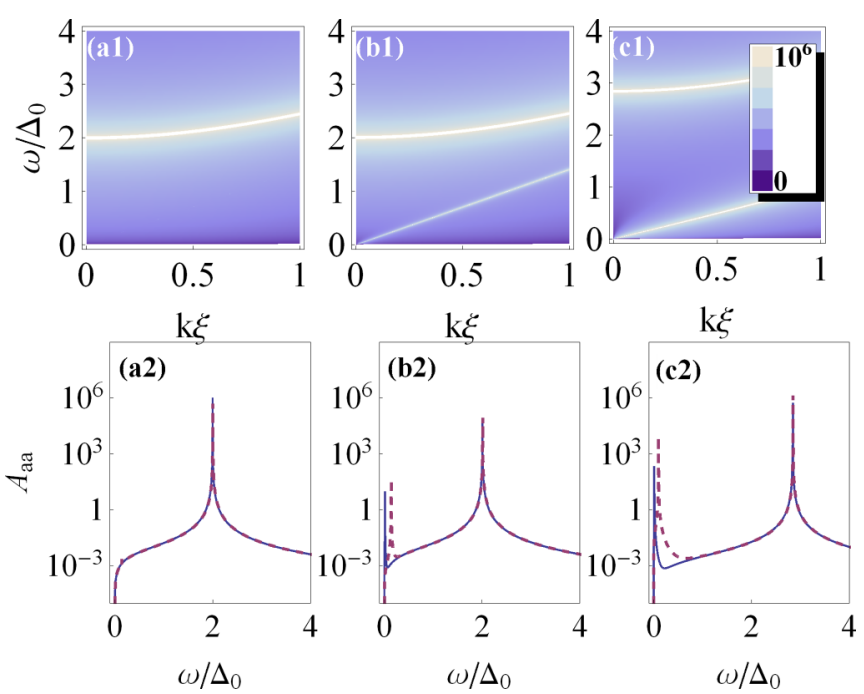

FIG. 2. Spectral function $A_{a a}(\mathbf{k}, \omega)$ in the absence of damping term. $A_{a a}(\mathbf{k}, \omega)$ as a function of $k$ (in units of $1 / \xi$ ) and $\omega$ (in units of $\left.\Delta_{0}\right)$ for three different interaction strengths $\zeta=-1 /\left(k_{F} a_{s}\right), \zeta=7$ for (a), $\zeta=3$ for (b), and $\zeta=1$ for (c), corresponding to different gaps $\Delta_{0} / E_{F}=10^{-5}, \Delta_{0} / E_{F}=4 \times 10^{-3}$, and $\Delta_{0} / E_{F}=7 \times 10^{-2}$, respectively. (a2)-(c2): $A_{a a}(\mathbf{k}, \omega)$ as a function of $\omega$ for $k=0.1 / \xi$ (purple dashed line) and $k=0.01 / \xi$ (blue solid line). $T / T_{c}=0.9$ and $\delta$ is taken as $10^{-4} \Delta_{0}$.

of freedom becomes stronger, as characterized by the offdiagonal term $i u^{\prime} \omega$ in Eq. (3). We note that for low-energy excitations such as the long-wavelength phase mode, this coupling is small $(\omega \rightarrow 0)$, but for the gapped Higgs mode, it provides significant mixing of the amplitude and phase. Such mixing is clearly illustrated in Figs. 2(a)-2(c), where we plot the spectral function $A_{a a}(\mathbf{k}, \omega)$ for three representative values of $\zeta$ (corresponding to different $\Delta_{0} / E_{\mathrm{F}}$ ). Two features can be noticed immediately. First, the Higgs gap increases beyond $2 \Delta_{0}$ of the BCS limit as the interaction strength increases. Second, there is increasing spectral weight transfer from the gapped Higgs mode to the gapless mode. One can, in fact, show explicitly that

$$
\frac{A_{+}}{A_{-}}=\frac{4 v^{\prime} r^{2}}{u^{\prime 2} \sqrt{k^{2} / 2 m^{*}\left(k^{2} / 2 m^{*}+2 r\right)}}
$$

which indicates the gradual increasing of the mixing between phase and amplitude degrees of freedom.

\section{EFFECTS OF DAMPING ON THE HIGGS MODE}

Due to the presence of the damping term, the timedependent Ginzburg-Landau theory is not a pure Lorentzinvariant $U(1)$ theory. Thus, at finite temperature, even in the BCS limit, the peak of Higgs excitation $\omega_{+}(\mathbf{k})$ will broaden as a result of damping. To calculate the equilibrium spectral weight in the presence of damping, we need to introduce the so-called Langevin force $\eta(t, \mathbf{x})$ [23], which satisfies the following conditions:

$$
\begin{array}{r}
\left\langle\eta\left(t^{\prime}, \mathbf{x}^{\prime}\right) \eta(t, \mathbf{x})\right\rangle=\left\langle\eta^{*}\left(t^{\prime}, \mathbf{x}^{\prime}\right) \eta^{*}(t, \mathbf{x})\right\rangle=0, \\
\left\langle\eta^{*}\left(t^{\prime}, \mathbf{x}^{\prime}\right) \eta(t, \mathbf{x})\right\rangle=2 u^{\prime \prime} k_{B} T \delta\left(t-t^{\prime}\right) \delta\left(\mathbf{x}-\mathbf{x}^{\prime}\right) .
\end{array}
$$
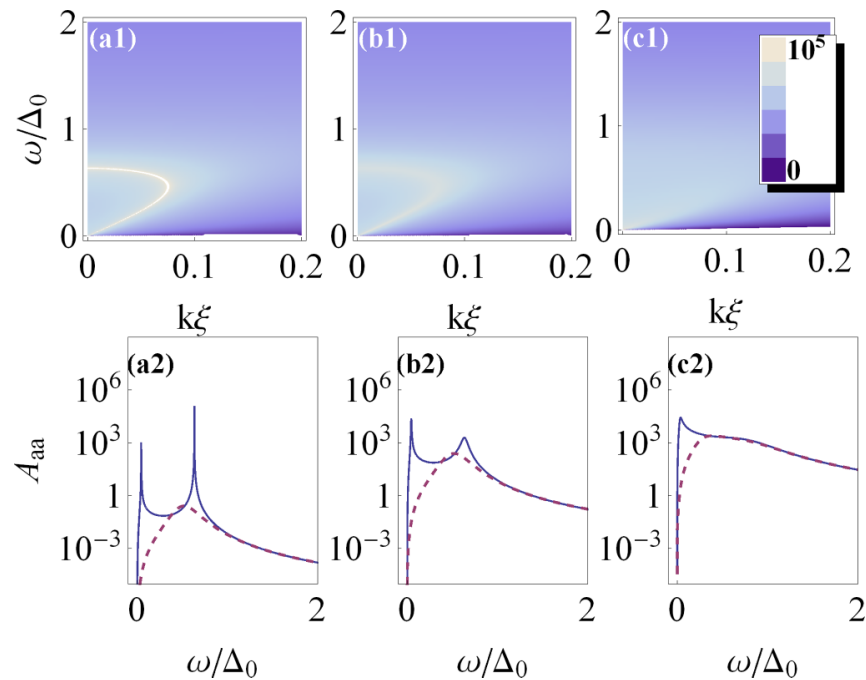

FIG. 3. Spectral function $A_{a a}(\mathbf{k}, \omega)$ in the presence of damping term. $A_{a a}(\mathbf{k}, \omega)$ as a function of $k$ (in unit of $1 / \xi$ ) and $\omega$ (in unit of $\left.\Delta_{0}\right)$ for three different interaction strengths $\zeta=-1 /\left(k_{F} a_{s}\right), \zeta=7$ for (a), $\zeta=3$ for (b), and $\zeta=1$ for (c), corresponding to different $\Delta_{0} / E_{F}=10^{-5}, \Delta_{0} / E_{F}=4 \times 10^{-3}$, and $\Delta_{0} / E_{F}=7 \times 10^{-2}$, respectively. (a2)-(c2): $A_{a a}(\mathbf{k}, \omega)$ as a function of $\omega$ for $k=0.1 / \xi$ (purple dashed line) and $k=0.01 / \xi$ (blue solid line). $T / T_{c}=0.2$ and $\delta$ are taken as $10^{-4} \Delta_{0}$.

Including the corresponding terms in the action as $S_{L}=$ $\int d t d^{3} \mathbf{x}\left(\phi^{*} \eta+\phi \eta^{*}\right)$, we obtain the equations of motion for $\delta_{a}$ and $\delta_{p}$ by setting $\partial\left(S+S_{L}\right) / \partial \delta_{a}=0$ and $\partial\left(S+S_{L}\right) /$ $\partial \delta_{p}=0$ :

$$
\begin{gathered}
\left(-v \omega^{2}+\xi_{\mathbf{k}}+2 r\right) \delta_{a}-i u \omega \delta_{p}+\eta^{\prime}=0, \\
\left(-v \omega^{2}+\xi_{\mathbf{k}}\right) \delta_{p}+i u \omega \delta_{a}+\eta^{\prime \prime}=0,
\end{gathered}
$$

where $\eta^{\prime}$ and $\eta^{\prime \prime}$ are the real and imaginary parts of the Langevin force $\eta$, respectively. The spectral functions for the amplitude fluctuation is given by using the fluctuation dissipation theorem [23],

$$
\begin{aligned}
A_{a a} & =\frac{u^{\prime \prime} \omega\left(\left|-v \omega^{2}+\frac{k^{2}}{2 m^{*}}\right|^{2}+|u \omega|^{2}\right)}{2\left|-(u \omega)^{2}+\left(-v \omega^{2}+\frac{k^{2}}{2 m^{*}}\right)\left(-v \omega^{2}+\frac{k^{2}}{2 m^{*}}+2 r\right)\right|^{2}} \\
& =\frac{u^{\prime \prime} \omega\left(\left|-v \omega^{2}+\frac{k^{2}}{2 m^{*}}\right|^{2}+|u \omega|^{2}\right)}{2\left|v^{\prime 2}\left(\omega^{2}-\tilde{\omega}_{+}^{2}\right)\left(\omega^{2}-\tilde{\omega}_{-}^{2}\right)-2 i u^{\prime} u^{\prime \prime} \omega^{2}\right|^{2}}
\end{aligned}
$$

where the eigenmode frequencies are given by

$$
\begin{aligned}
\tilde{\omega}_{ \pm}^{2}= & \frac{\xi_{\mathbf{k}}+r}{v^{\prime}}+\frac{u^{\prime 2}-u^{\prime \prime 2}}{2 v^{\prime 2}} \\
& \pm \sqrt{\frac{r^{2}}{v^{\prime 2}}+\frac{\left(u^{\prime 2}-u^{\prime 2}\right)^{2}}{4 v^{\prime 4}}+\frac{u^{\prime 2}-u^{\prime 2}}{v^{\prime 3}}\left(\xi_{\mathbf{k}}+r\right) .}
\end{aligned}
$$

By comparing Fig. 3 (with damping) with Fig. 2 (without damping), one can see three important features brought about by the damping term:

(i) The spectral weight transfer is enhanced. For instance, for $\zeta=-7$, there is almost no spectral weight transfer in the absence of damping [Fig. 2(a)], while in the presence 
of damping, for very small $k \ll 1 / \xi, A_{a a}(\mathbf{k}, \omega)$ exhibits a clear peak at the energy of the phase mode. For small $\xi_{\mathbf{k}}$ the spectral weight on the phase mode can be calculated as $A_{a a}\left(\tilde{\omega}_{-}, \mathbf{k}\right) \simeq u^{\prime \prime} / 8 u^{\prime 2} \tilde{\omega}_{-}$. Different from the case without a damping term, we see that in the case with a damping term the spectral function has a weight proportional to $u^{\prime \prime}$ on the phase mode. A similar enhancement of spectral weight transfer can also be easily seen in Fig. 3(b) for $\zeta=-3$.

(ii) For $k \ll 1 / \xi$, the eigenmode frequencies can be approximated as

$$
\begin{aligned}
& \tilde{\omega}_{-}=\sqrt{\frac{2 r \xi_{\mathbf{k}}}{2 v^{\prime} r+u^{\prime 2}-u^{\prime \prime 2}}}, \\
& \tilde{\omega}_{+}=\sqrt{\frac{2 v^{\prime} r+u^{\prime 2}-u^{\prime \prime 2}}{v^{\prime 2}}+\frac{2 v^{\prime} r+2 u^{\prime 2}-2 u^{\prime \prime 2}}{v^{\prime}\left(2 v^{\prime} r+u^{\prime 2}-u^{\prime \prime 2}\right)} \xi_{\mathbf{k}} .}
\end{aligned}
$$

It is straightforward to see that at the BCS limit the location of the Higgs peak is substantially reduced from $\sqrt{2 r / v^{\prime}}$ to $\sqrt{2 r / v^{\prime}-u^{\prime \prime 2} / v^{\prime 2}}$, as shown for $\zeta=-7$ and -3 in Figs. 3(a) and 3(b), respectively.

(iii) As $k$ starts to deviate from zero, the Higgs mode quickly loses its identity due to strong hybridization with the phase mode. For instance, even for $k=0.1 / \xi$, as displayed by the purple dashed line in Figs. 3(a2) to 3(c2), no sharp peak feature is observed in $A_{a a}(\mathbf{k}, \omega)$. And for $\zeta=-1$, no sharp peak exists even for $k=0.01 / \xi$.

\section{CONCLUSIONS}

In summary, we have investigated the evolution of the Higgs mode in the BEC-BCS crossover for a neutral Fermi superfluid. A time-dependent Ginzburg-Landau (TDGL) theory with a second-order time derivative is derived. By computing explicitly the parameters in the TDGL theory with NSR, it shows how the theory gradually loses its Lorentz invariance and changes from a relativistic theory in the BCS side to a nonrelativistic theory with Galilean invariance in the BEC limit. We further include the damping terms in the TDGL theory, which reflects the composite nature of the pairing field $\phi$.

Within the TDGL formulation and coupled with microscopic NSR calculation, we obtain the following conclusions regarding the appearance and characterization of the Higgs mode:

(i) In the BCS limit, the existence of the Higgs mode is guaranteed by the Lorentz invariance. Towards the unitary regime and BEC side, as the system gradually loses the Lorentz invariance, the Higgs mode is pushed to very high energy and its spectral weight is increasingly transferred to the phase mode.

(ii) On the BCS side, a damping term arises due to coupling between the Cooper pair field and the fermionic quasiparticles. This damping term strongly couples the Higgs mode to the gapless phase mode in the neutral superfluid, which enhances the spectral weight transfer and washes out features of the Higgs mode, especially at finite momentum.

\section{ACKNOWLEDGMENTS}

We thank Georg Bruun for very useful discussions. B.Y. and H.Z. are supported by the Tsinghua University Initiative Scientific Research Program, NSFC Grants No. 11174176 and No. 11325418, and the NKBRSFC under Grant No. 2011CB921500. S.Z. is supported by a start-up grant from the University of Hong Kong, the Collaborative Research Fund (Grants No. HKUST3/CRF/13G and No. RGC-GRF 17306414), and the Croucher Foundation under the Croucher Innovation Award. H.Z. would like to thank Hong Kong University for hospitality, where this work was initiated.

\section{APPENDIX: TIME-DEPENDENT GINZBURG-LANDAU THEORY}

A time-dependent Ginzburg-Landau theory can be constructed for the entire BEC-BCS crossover in the vicinity of $T_{\mathrm{c}}[21,24]$. The partition function takes the form $\mathcal{Z}=$ $\int D\left[\bar{\psi}_{\sigma}, \psi_{\sigma}\right] e^{-S\left[\bar{\psi}_{\sigma}, \psi_{\sigma}\right]}$, with

$$
\begin{aligned}
S\left[\bar{\psi}_{\sigma}, \psi_{\sigma}\right]= & \int d \tau d^{3} \mathbf{x}\left\{\bar{\psi}_{\sigma}\left(\partial_{\tau}-\frac{\nabla^{2}}{2 m}-\mu\right) \psi_{\sigma}\right. \\
& \left.-g \bar{\psi}_{\uparrow} \bar{\psi}_{\downarrow} \psi_{\downarrow} \psi_{\uparrow}\right\},
\end{aligned}
$$

where $\psi_{\sigma}$ are Grassmann fields and $g$ is the contact interaction between fermions of opposite spins. $\mu$ is the chemical potential, which is determined by requiring the number density to be equal to $n$. To investigate the fluctuation effects in the Cooper channel, we use a Hubbard-Stratonovich transformation to decouple the interaction term in the Cooper channel and then integrating out the fermions. We obtain an effective theory for the bosonic field $\Delta(\tau, \mathbf{x})$, which represents the Cooper pair field. Straightforward calculations yield the partition function in terms of field $\Delta$ as

$$
\mathcal{Z}=\int D(\bar{\Delta}, \Delta) \exp \left[-\frac{1}{g} \int d \tau d \mathbf{x}|\Delta|^{2}+\ln \operatorname{det} \hat{G}^{-1}\right]
$$

where

$$
\hat{G}^{-1}=\left(\begin{array}{cc}
-\partial_{\tau}+\frac{\nabla^{2}}{2 m}+\mu & \Delta \\
\bar{\Delta} & -\partial_{\tau}-\frac{\nabla^{2}}{2 m}-\mu
\end{array}\right)
$$

is the Gorkov Green function.

In the vicinity of the phase transition, the gap parameter $\Delta$ is small and an expansion in terms of $\Delta$ becomes possible. Including both the spatial and time derivatives (after Wick rotation) and retaining the parameter $\Delta$ up to the fourth order we obtain an effective action as

$$
S[\bar{\Delta}, \Delta]=\int d t d^{3} \mathbf{x}\left\{\bar{\Delta}\left[-i u \partial_{t}+v \partial_{t}^{2}-\frac{\nabla^{2}}{2 m^{*}}-r\right] \Delta+\frac{b}{2} \bar{\Delta} \bar{\Delta} \Delta \Delta\right\},
$$


where $u=u^{\prime}+i u^{\prime \prime}$ and $v=v^{\prime}+i v^{\prime \prime}$ are complex in general and all the parameters can be expressed in terms of microscopic parameters as

$$
\begin{aligned}
& u^{\prime}=\frac{(2 m)^{3 / 2}}{16 \pi^{2}}\left[\frac{2 \sqrt{2} \beta \sqrt{|\mu|}}{\pi} \sum_{n=0}^{\infty} \frac{\sqrt{\sqrt{1+(2 n+1)^{2}\left(\frac{\pi}{\beta \mu}\right)^{2}}-\operatorname{sgn}(\mu)}}{(2 n+1)^{2}}-\frac{\pi \beta}{2} \sqrt{|\mu|} \theta(-\mu)\right] \\
& u^{\prime \prime}=\frac{m^{3 / 2}}{8 \sqrt{2} \pi} \beta \sqrt{|\mu|} \Theta(\mu), \\
& v^{\prime}=\frac{(2 m)^{3 / 2}}{32 \pi^{2}}\left[\frac{2 \sqrt{2} \beta^{2} \sqrt{|\mu|}}{\pi^{2}} \sum_{n=0}^{\infty} \frac{\sqrt{\sqrt{1+(2 n+1)^{2}\left(\frac{\pi}{\beta \mu}\right)^{2}}+\operatorname{sgn}(\mu)}}{(2 n+1)^{3}}-\frac{\pi \beta}{4 \sqrt{|\mu|}} \theta(-\mu)\right], \\
& v^{\prime \prime}=-\frac{m^{3 / 2}}{32 \sqrt{2} \pi} \frac{\beta}{\sqrt{|\mu|}} \Theta(\mu), \\
& \frac{1}{2 m^{*}}=\frac{1}{2 m} \int \frac{d^{3} \mathbf{k}}{(2 \pi)^{3}}\left\{\frac{1-2 N\left(\xi_{\mathbf{k}}\right)}{8 \xi_{\mathbf{k}}^{2}}+\frac{\frac{\partial N\left(\xi_{\mathbf{k}}\right)}{\partial \xi_{\mathbf{k}}}}{4 \xi_{\mathbf{k}}}+\frac{\frac{\partial^{2} N\left(\xi_{\mathbf{k}}\right)}{\partial \xi_{\mathbf{k}}^{2}} \cdot \frac{\mathbf{k}^{2}}{2 m}}{6 \xi_{\mathbf{k}}}\right\}, \\
& r=\frac{m}{4 \pi a}+\int \frac{d^{3} \mathbf{k}}{(2 \pi)^{3}}\left\{\frac{1-2 N\left(\xi_{\mathbf{k}}\right)}{2 \xi_{\mathbf{k}}}-\frac{1}{2 \epsilon_{\mathbf{k}}}\right\}, \\
& b=\int \frac{d^{3} \mathbf{k}}{(2 \pi)^{3}}\left\{\frac{1-2 N\left(\xi_{\mathbf{k}}\right)}{4 \xi_{\mathbf{k}}^{3}}+\frac{\beta N\left(\xi_{\mathbf{k}}\right)\left[N\left(\xi_{\mathbf{k}}\right)-1\right]}{2 \xi_{\mathbf{k}}^{2}}\right\} .
\end{aligned}
$$

In the above equations, $N\left(\xi_{\mathbf{k}}\right)=1 /\left[\exp \left(\beta \xi_{\mathbf{k}}\right)+1\right]$ is the Fermi distribution function and $\xi_{\mathbf{k}}=\epsilon_{\mathbf{k}}-\mu$ with $\epsilon_{\mathbf{k}}=\mathbf{k}^{2} / 2 m$. Function $\Theta(2 \mu)$ is the heaviside step function. Explicitly, the parameter $b$ is the result of a one-loop calculation with four fermion propagators:

$$
b=-\frac{1}{\beta^{2}} \sum_{\omega_{n}} \int \frac{d^{3} \mathbf{k}}{(2 \pi)^{3}} \frac{1}{\left(-i \omega_{n}+k^{2} / 2 m-\mu\right)^{2}} \frac{1}{\left(i \omega_{n}+k^{2} / 2 m-\mu\right)^{2}} .
$$

The other parameters $u, v, \frac{1}{2 m^{*}}$, and $r$ are all derived from the inverse vertex function $\Gamma^{-1}\left(\omega_{n}, \mathbf{k}\right)$, which after the standard renormalization by replacing $g$ with the two-body scattering length $a_{\mathrm{s}}$ is given by

$$
\Gamma^{-1}\left(\omega_{n}, \mathbf{k}\right)=-\frac{m}{4 \pi a_{\mathrm{s}}}-\int \frac{d^{3} \mathbf{q}}{(2 \pi)^{3}}\left\{\frac{1-N\left(\epsilon_{\mathbf{q}}-\mu\right)-N\left(\epsilon_{\mathbf{k}-\mathbf{q}}-\mu\right)}{-i \omega_{n}+\epsilon_{\mathbf{q}}+\epsilon_{\mathbf{k}-\mathbf{q}}-2 \mu}-\frac{1}{2 \epsilon_{\mathbf{q}}}\right\} .
$$

To derive the time-dependent Ginzburg-Landau equation, we first analytically continue the vertex function to real frequency $i \omega_{n} \rightarrow \omega+i 0^{+}$. This procedure generates a time-dependent term with the parameters $u$ and $v$. The detailed derivation is as follows:

The frequency-dependent part of $\Gamma^{-1}(\omega, \mathbf{k})$ is

$$
\Gamma^{-1}(\omega, 0)-\Gamma^{-1}(0,0)=-\frac{m}{4 \pi a}-\int \frac{d^{3} \mathbf{k}}{(2 \pi)^{3}}\left\{\frac{1-2 N\left(\epsilon_{\mathbf{k}}-\mu\right)}{-\omega-i \eta+2 \epsilon_{\mathbf{k}}-2 \mu}-\frac{1}{2 \epsilon_{\mathbf{k}}}\right\}-\Gamma^{-1}(0,0) .
$$

Then we expand it in a series of small $\omega$ as

$$
\Gamma^{-1}(\omega, 0)-\Gamma^{-1}(0,0) \simeq-\omega \int \frac{d^{3} \mathbf{k}}{(2 \pi)^{3}} \frac{1-2 N\left(\epsilon_{\mathbf{k}}-\mu\right)}{\left(2 \epsilon_{\mathbf{k}}-2 \mu-i \eta\right)^{2}}-\omega^{2} \int \frac{d^{3} \mathbf{k}}{(2 \pi)^{3}} \frac{1-2 N\left(\epsilon_{\mathbf{k}}-\mu\right)}{\left(2 \epsilon_{\mathbf{k}}-2 \mu-i \eta\right)^{3}} .
$$

We define the parameters as $u \equiv \int \frac{d^{3} \mathbf{k}}{(2 \pi)^{3}} \frac{1-2 N\left(\epsilon_{\mathbf{k}}-\mu\right)}{\left(2 \epsilon_{\mathbf{k}}-2 \mu-i \eta\right)^{2}}$ and $v \equiv \int \frac{d^{3} \mathbf{k}}{(2 \pi)^{3}} \frac{1-2 N\left(\epsilon_{\mathbf{k}}-\mu\right)}{\left(2 \epsilon_{\mathbf{k}}-2 \mu-i \eta\right)^{3}}$. They both can be calculated by contour integration,

$$
\begin{aligned}
u & \equiv \int \frac{d^{3} \mathbf{k}}{(2 \pi)^{3}} \frac{1-2 N\left(\epsilon_{\mathbf{k}}-\mu\right)}{\left(2 \epsilon_{\mathbf{k}}-2 \mu-i \eta\right)^{2}}=\frac{v\left(\epsilon_{F}\right)}{4 \sqrt{\epsilon_{F}}} \int_{0}^{\infty} d \epsilon \sqrt{\epsilon} \frac{1-2 N(\epsilon-\mu)}{(\epsilon-\mu-i \eta)^{2}} \\
& =\frac{v\left(\epsilon_{F}\right)}{4 \sqrt{\epsilon_{F}}} \frac{1}{2} \int_{c} d z \sqrt{z} \frac{1-2 N(z-\mu)}{(z-\mu-i \eta)^{2}}
\end{aligned}
$$




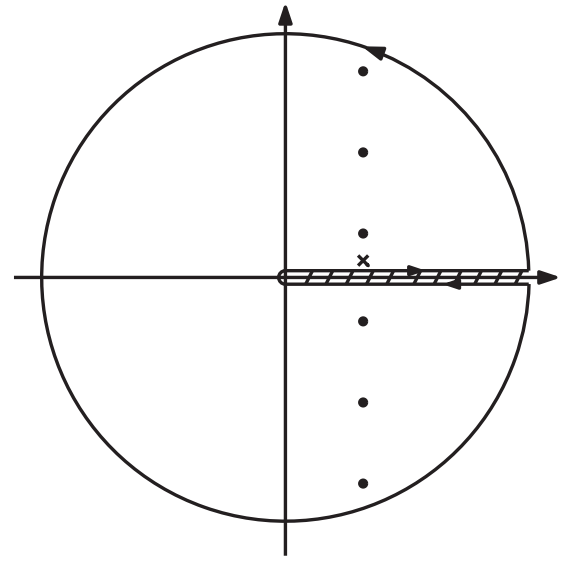

FIG. 4. The contour $\mathrm{c}$ in the calculation of Eq. (A16). The dots (.) denote the first-order poles $z_{n}=\mu+\frac{(2 n+1) \pi i}{\beta}$ and the cross $(\times)$ denotes the second-order pole $z_{\eta}=\mu+\eta i$.
TABLE I. Asymptotic behaviors of the parameters in the timedependent Ginzburg-Landau theory in the BCS and BEC limits.

\begin{tabular}{lcc}
\hline \hline Parameters & BCS limit & BEC limit \\
\hline$u^{\prime}$ & 0 & $\frac{\pi v\left(\epsilon_{F}\right)}{8 \sqrt{\epsilon_{F}|\mu|}}$ \\
$u^{\prime \prime}$ & $v\left(\epsilon_{F}\right) \frac{\pi}{8 k_{B} T}$ & 0 \\
$v^{\prime}$ & $\frac{7 v\left(\epsilon_{F}\right)}{16 \pi^{2}\left(k_{B} T\right)^{2}} \zeta(3)$ & $\frac{\pi v\left(\epsilon_{F}\right)}{64 \sqrt{\epsilon_{F}}|\mu|^{3 / 2}}$ \\
$v^{\prime \prime}$ & $-v\left(\epsilon_{F}\right) \frac{\pi}{32 k_{B} T \epsilon_{F}}$ & 0 \\
$\frac{1}{2 m^{*}}$ & $\frac{1}{2 m} \frac{7 v\left(\epsilon_{F}\right) \epsilon_{F}}{12 \pi^{2}\left(k_{B} T\right)^{2}} \zeta(3)$ & $\frac{1}{2 m} \frac{\pi v\left(\epsilon_{F}\right)}{16 \sqrt{\epsilon_{F}}|\mu|}$ \\
$r$ & $v\left(\epsilon_{F}\right) \ln \frac{T_{C}}{T}$ & $\frac{\pi v\left(\epsilon_{F}\right)}{2 \sqrt{2} \sqrt{\epsilon_{F}}}\left(\frac{1}{\sqrt{m} a_{s}}-\sqrt{2|\mu|}\right)$ \\
$b$ & $\frac{7 v\left(\epsilon_{F}\right)}{8 \pi^{2}\left(k_{B} T\right)^{2}} \zeta(3)$ & $\frac{\pi v\left(\epsilon_{F}\right)}{32 \sqrt{\epsilon_{F}}|\mu|^{3 / 2}}$ \\
\hline \hline
\end{tabular}

where $c$ denotes the contour in Fig. 4. There are infinite first-order poles $z_{n}=\mu+\frac{(2 n+1) \pi i}{\beta}$ and one second-order pole $z_{\eta}=\mu+\eta i$.

The contour integration can be evaluated in the summation of the residuals as

$$
\begin{aligned}
\int_{c} d z \sqrt{z} \frac{1-2 N(z-\mu)}{(z-\mu-i \eta)^{2}} & =2 \pi i\left\{\sum_{n=-\infty}^{+\infty} \lim _{z \rightarrow z_{n}} \frac{2 \sqrt{z} / \beta}{(z-\mu-\eta i)^{2}}+\lim _{z \rightarrow z_{\eta}} \frac{d}{d z}\left[\sqrt{z}\left(1-\frac{2}{\exp [\beta(z-\mu)]+1}\right)\right]\right\} \\
& =2 \pi i\left[-\frac{2 \beta \sqrt{\mu}}{\pi^{2}} \sum_{n=-\infty}^{+\infty} \frac{\sqrt{1+(2 n+1) \pi i / \beta \mu}}{(2 n+1)^{2}}+\sqrt{\mu} \frac{\beta}{2}\right] .
\end{aligned}
$$

Calculation shows that $\sum_{n=-\infty}^{+\infty} \frac{\sqrt{1+(2 n+1) \pi i / \beta \mu}}{(2 n+1)^{2}}$ is purely imaginary due to the symmetry of the $z_{n}$ pole locations with respect to the horizontal axes. Then it can be written as $\sum_{n=-\infty}^{+\infty} \frac{\sqrt{1+(2 n+1) \pi i / \beta \mu}}{(2 n+1)^{2}}=\sqrt{2} i \sum_{n=0}^{+\infty} \frac{\sqrt{\sqrt{1+[(2 n+1) \pi / \beta \mu]^{2}}-\operatorname{sgn}(\mu)}}{(2 n+1)^{2}}$. Hence, the parameter $u$ is calculated as

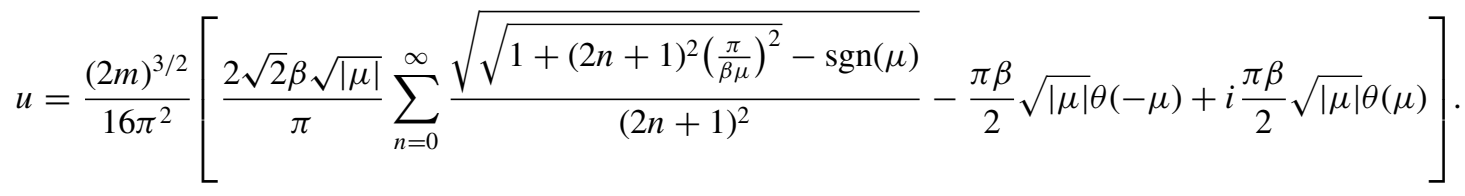

In the same manner, the parameter $v$ can also be calculated as shown in Eqs. (6) and (7). We should note that while the expressions for $u$ and others look different from the standard expression, as given in Ref. [24], they in fact reduce to the same expressions. We found that this form is more convenient to use the above expression when dealing with higher-order time-derivative terms.

In the BCS and BEC limits all the parameters can be analytically derived as shown in Table I.

[1] CMS Collaboration, Phys. Lett. B 716, 30 (2012).

[2] ATLAS Collaboration, Phys. Lett. B 716, 1 (2012).

[3] R. Sooryakumar and M. V. Klein, Phys. Rev. Lett. 45, 660 (1980).

[4] P. B. Littlewood and C. M. Varma, Phys. Rev. B 26, 4883 (1982).

[5] P. B. Littlewood and C. M. Varma, Phys. Rev. Lett. 47, 811 (1981).

[6] Ch. Rüegg, B. Normand, M. Matsumoto, A. Furrer, D. F. McMorrow, K. W. Kramer, H. U. Gudel, S. N. Gvasaliya, H. Mutka, and M. Boehm, Phys. Rev. Lett. 100, 205701 (2008).

[7] R. Matsunaga, Y. I. Hamada, K. Makise, Y. Uzawa, H. Terai, Z. Wang, and R. Shimano, Phys. Rev. Lett. 111, 057002 (2013).

[8] R. Matsunaga, N. Tsuji, H. Fujita, A. Sugioka, K. Makise, Y. Uzawa, H. Terai, Z. Wang, H. Aoki, and R. Shimano, Science 345, 1145 (2014).
[9] U. Bissbort, S. Götze, Y. Li, J. Heinze, J. S. Krauser, M. Weinberg, C. Becker, K. Sengstock, and W. Hofstetter, Phys. Rev. Lett. 106, 205303 (2011).

[10] M. Endres, T. Fukuhara, D. Pekker, M. Cheneau, P. Schaub, C. Gross, E. Demler, S. Kuhr, and I. Bloch, Nature (London) 487, 454 (2012).

[11] C. M. Varma, J. Low Temp. Phys. 126, 901 (2002).

[12] Y. Barlas and C. M. Varma, Phys. Rev. B 87, 054503 (2013).

[13] S. D. Huber, B. Theiler, E. Altman, and G. Blatter, Phys. Rev. Lett. 100, 050404 (2008).

[14] L. Pollet and N. Prokof'ev, Phys. Rev. Lett. 109, 010401 (2012).

[15] D. Pekker and C. M. Varma, Annu. Rev. Condens. Matter Phys. 6, 269 (2015). 
[16] D. Podolsky, A. Auerbach, and D. P. Arovas, Phys. Rev. B 84, 174522 (2011).

[17] D. Podolsky and S. Sachdev, Phys. Rev. B 86, 054508 (2012).

[18] S. Gazit, D. Podolsky, and A. Auerbach, Phys. Rev. Lett. 110, 140401 (2013).

[19] A. Rançon and N. Dupuis, Phys. Rev. B 89, 180501(R) (2014).

[20] G. M. Bruun, Phys. Rev. A 90, 023621 (2014).
[21] M. Randeria, in Bose-Einstein Condensation, edited by A. Griffin, D. W. Snokes, and S. Stringari (Cambridge University Press, Cambridge, UK, 1995).

[22] P. Nozières and S. Schmitt-Rink, J. Low Temp. Phys. 59, 195 (1985).

[23] M. Tinkham, Introduction to Superconductivity, 2nd ed. (Dover Publications, New York, 2004).

[24] C. A. R. Sá de Melo, M. Randeria, and J. R. Engelbrecht, Phys. Rev. Lett. 71, 3202 (1993). 